\title{
Genre et vie de couple au cours de la maladie à VIH
}

\author{
France LeRT ${ }^{1,2}$, Rémi SITTA ${ }^{1,2}$, Morgan HAMON ${ }^{1,2}$, Rosemary DRAY-SPIRA ${ }^{1,2}$, \\ Anne-Déborah BoUHNIK ${ }^{3}$, Bruno SPIRE ${ }^{3}$ et le groupe Anrs-Vespa
}

\section{Résumé}

Le maintien ou la construction d'une relation de couple constitue une ressource identitaire pour faire face à l'infection à VIH et un soutien fort pour faire face à la maladie. Si en population générale, à l'âge adulte, la vie en couple constitue le cadre principal de la vie affective et sexuelle pour les hétérosexuels, chez les homosexuels, il n'est pas le mode dominant, en dépit d'une augmentation, puis une stabilisation survenue dans la mouvance des changements profonds suscités par le sida dans les deux dernières décennies.

Sur la base des données de l'enquête Vespa réalisée en 2003, cet article porte sur les déterminants démographiques, sociaux, comportementaux et médicaux de deux modalités de la vie de couple, avec et sans cohabitation, chez les femmes hétérosexuelles, les hommes, homo, bi ou hétérosexuels, soit 2900 personnes. Chez les hétérosexuels, la progression de la maladie et la perte du conjoint se marquent dans une absence de vie de couple. Chez les homosexuels, celle-ci apparaît plutôt comme un style de vie « choisi » en regard d'une vie sexuelle marquée par un nombre élevé de partenaires. A la différence des femmes et des homosexuels masculins, les hommes hétérosexuels ont plus de chances de maintenir une vie de couple en avançant en âge. Le profil des personnes en couple sans cohabitation se rapproche de celui des personnes seules.

Mots clés : relation de couple, hétérosexuels, homosexuels, femmes.

L'infection par le VIH reste, malgré les traitements très efficaces, une maladie grave qui entrave les projets personnels pour de nombreuses personnes atteintes. Ce poids est particulièrement marqué dans le domaine affectif et sexuel : c'est en effet dans la sexualité que l'infection se rappelle en permanence par la menace de la transmission, comme un obstacle à la spontanéité de l'élan amoureux et sexuel [1]. Certaines personnes font l'expérience directe d'un rejet ou d'attitudes négatives de la part de leur partenaire mais, de façon plus insidieuse, la qualité de la relation de couple peut être altérée par le ressentiment, la frustration ou le sentiment de la personne atteinte de ne pas être en mesure de satisfaire les besoins ou les attentes de son partenaire. Souvent, l'infection est également présente chez l'autre

Unité Inserm 687, Saint-Maurice.

IFR 69 , Villejuif.

Unité Inserm 379-0bservatoire régional de la santé Paca, Marseille. partenaire du couple. Si cette condition partagée peut constituer un soutien, elle peut aussi être porteuse d'une inquiétude supplémentaire pour l'autre, confronté à l'aggravation de la maladie ou en redoutant la survenue. Enfin, nombre d'entre eux ont été confrontés à la mort prématurée de leurs conjoints ou partenaires, surtout dans les générations plus anciennes ou parmi ceux qui viennent de pays où les traitements ne sont pas disponibles.

La transmission sexuelle du virus a conduit à mettre l'accent, dans la recherche comme dans le discours préventif, sur les comportements sexuels, sur l'acte sexuel et les facteurs associés aux pratiques préventives au détriment de la dimension affective et sociale de la sexualité qui contribue à en faire une ressource identitaire, sociale et économique pour vivre et faire face à la maladie [2]. Si la vie en couple constitue le cadre principal de la sexualité pour les hétérosexuels, chez les homosexuels masculins, l'ère du sida a entraîné des changements dans les modes de vie, avec, au début des années 1990, un mouvement en faveur de la constitution du couple, mouvement qui semble maintenant stabilisé $[3,4]$. L'existence d'une relation stable a été trouvée associée à une évolution plus favorable de l'infection à VIH $[5,6]$

L'objet de cette analyse est de décrire la vie de couple chez les personnes vivant avec le VIH, en étudiant la part des facteurs démographiques, sociaux, et comportementaux et des facteurs liés à la maladie.

\section{Matériel et méthode}

L'analyse porte sur les données de l'enquête Vespa, réalisée en 2003, sur un échantillon national de consultations hospitalières auprès de personnes, majeures, diagnostiquées pour leur séropositivité depuis au moins 6 mois. Ces personnes ont répondu à un questionnaire passé en face à face d'une durée de 45 minutes environ et couvrant un large éventail d'informations sur la vie quotidienne. La méthodologie et le protocole de l'enquête sont détaillés page 215 [7].

Notre étude de la vie de couple a été réalisée en fonction du sexe et de l'autodéfinition de la personne comme homo, bi ou hétérosexuelle afin de saisir les logiques à l'œuvre dans chaque groupe. Les femmes homo ou bisexuelles et les transgenres n'ont pas été 
Figure 1

Mode de vie selon l'identité sexuelle déclarée $(n=2900)$

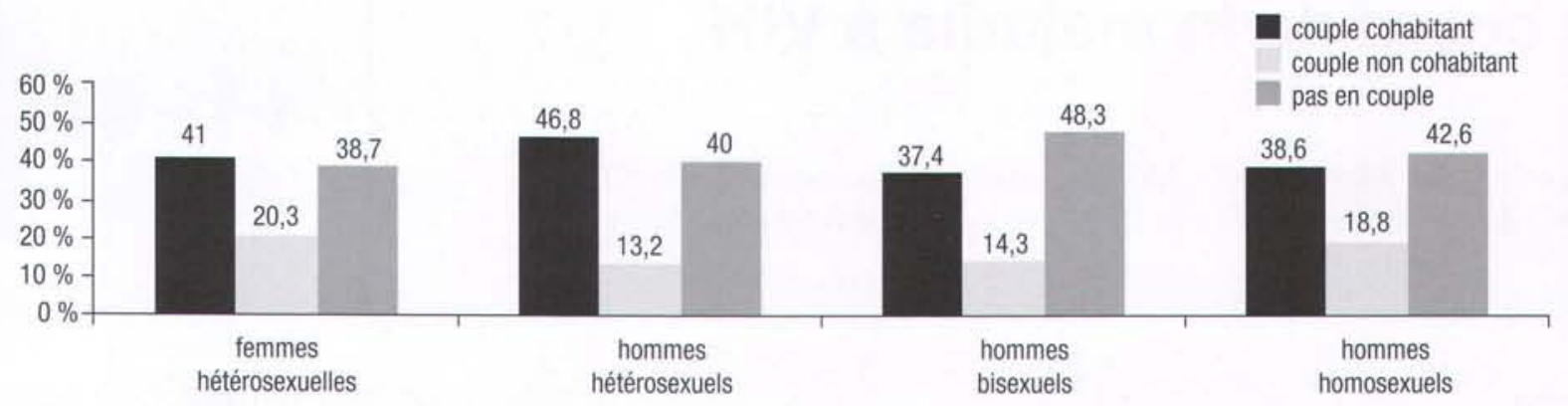

étudiés en raison de leur petit nombre (28 personnes). Les participants à l'enquête étaient invités à dire s'ils avaient une vie de couple, qu'ils habitent ensemble ou non, la notion de couple étant laissée à leur appréciation sans repère de durée (la cohabitation était documentée séparément). Quatre personnes ont donné des informations incohérentes et ont été exclues de l'analyse qui porte sur 2900 personnes.

La variable étudiée pour rendre compte de la situation du couple comporte trois modalités : couple cohabitant, couple non cohabitant, non en couple ou seul. Pour les bisexuels, s'ajoute pour certaines analyses une distinction selon le sexe du partenaire dans la relation de couple. Pour l'année en cours, une seule relation de couple donnait lieu à une information détaillée.

En premier lieu, la situation de couple est décrite en relation avec les caractéristiques démographiques (âge, lieu de naissance, région de résidence), les variables sociales (niveau d'éducation et importance accordée à la religion), le comportement sexuel au cours de la vie (le nombre de partenaires du même sexe pour les homo et bisexuels masculins, pour les hétérosexuels, l'existence de partenaires du même sexe au cours de la vie et le nombre de partenaires de sexe opposé) et l'existence d'un usage de drogue intraveineux ancien ou encore actuel. Concernant la maladie, ont été pris en compte l'ancienneté du diagnostic, le stade sida et un indicateur de survenue d'un épisode sévère depuis le diagnostic. Cet indicateur dichotomique est construit à partir des quelques informations disponibles dans le questionnaire médical ; sont considérés comme ayant une forte probabilité d'un épisode sévère, les patients diagnostiqués au stade sida ou avec moins de $200 \mathrm{CD} 4$ ou mis sous traitement à moins de $350 \mathrm{CD} 4^{4}$. Enfin le décès d'un (e) conjoint(te)/compagnon(gne) du sida est également étudié.

Toutes les analyses ont été faites séparément pour chacun des quatre groupes: femmes hétérosexuelles, hommes hétérosexuels, homosexuels masculins, bisexuels masculins. Pour chaque groupe (sauf celui des hommes bisexuels en raison d'effectifs insuffisants), on a comparé séparément les couples coha-

\footnotetext{
4 On fait l'hypothèse que, dans un contexte d'accès aisé aux traitements, les patients qui n'ont pas de traitement et ceux pour lesquels le diagnostic n'a pas été tardif n'ont pas connu de déficit immunitaire. Le stade sida est pris en compte spécifiquement.
}

bitants puis les couples non cohabitants aux personnes ne vivant pas en couple. Cette distinction tient à ce que l'on a considéré que la cohabitation était la norme chez les hétérosexuels autour de 40 ans, alors que la relation stable sans cohabitation était plutôt une situation transitoire ou minoritaire. Toutes les analyses ont été réalisées sur des données pondérées pour tenir compte du plan de sondage et de la participation [8].

\section{Résultats}

Les 2900 répondants de l'enquête se répartissaient en 774 femmes et 916 hommes hétérosexuels, 211 bisexuels et 999 homosexuels. La situation au regard de la vie de couple au moment de l'enquête est représentée sur la figure 1. La vie en couple cohabitant est la modalité la plus fréquente chez les femmes et les hommes hétérosexuels (respectivement $41 \%$ et $46,8 \%$ ), tandis que chez les hommes bisexuels et homosexuels, l'absence de relation de couple est la situation la plus fréquente (respectivement $48,3 \%$ et $42,6 \%$ ).

Le tableau 1 montre que la proportion de femmes vivant en couple cohabitant diminue après 40 ans, diminue avec l'importance de la religion, et est plus faible en île-de-France par rapport aux autres régions et chez celles de très bas niveau d'étude. Les femmes originaires d'Afrique sub-saharienne tendent à vivre moins souvent en couple que les Françaises de naissance. Les femmes dont le diagnostic a été posé depuis 2000 et celles qui sont au stade sida vivent moins souvent en couple. La cohabitation n'est associée ni au nombre de partenaires masculins dans la vie, ni au fait d'avoir eu des partenaires féminines. La perte d'un conjoint du sida est fortement associée à un veuvage lié au sida (tableau 1). En analyse multivariée, l'âge supérieur à 50 ans, la résidence en Île-de-France, le stade sida, le fait d'être encore substituée ou consommatrice de drogues et d'avoir perdu un conjoint, sont associés à un probabilité plus faible de vivre en cohabitation; on observe également une association positive entre le niveau d'étude et la vie en couple (tableau 1)

Le fait d'avoir un partenaire stable sans cohabiter, comparé à l'absence de vie de couple, est plus fréquent en région, diminue avec l'âge et est moins fréquent chez les femmes dépistées avant 


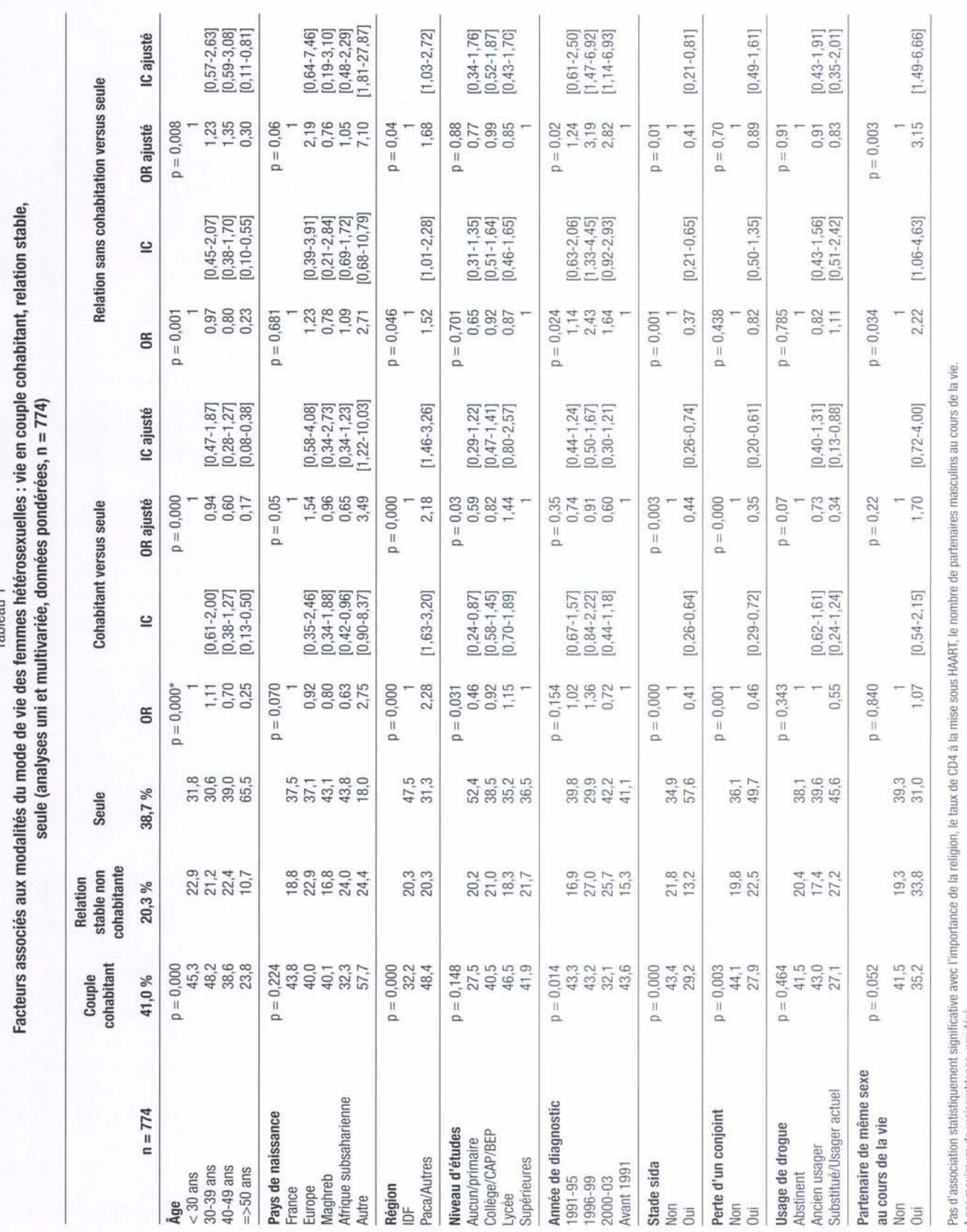

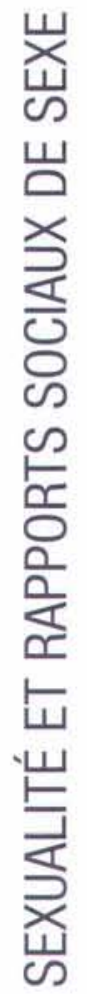




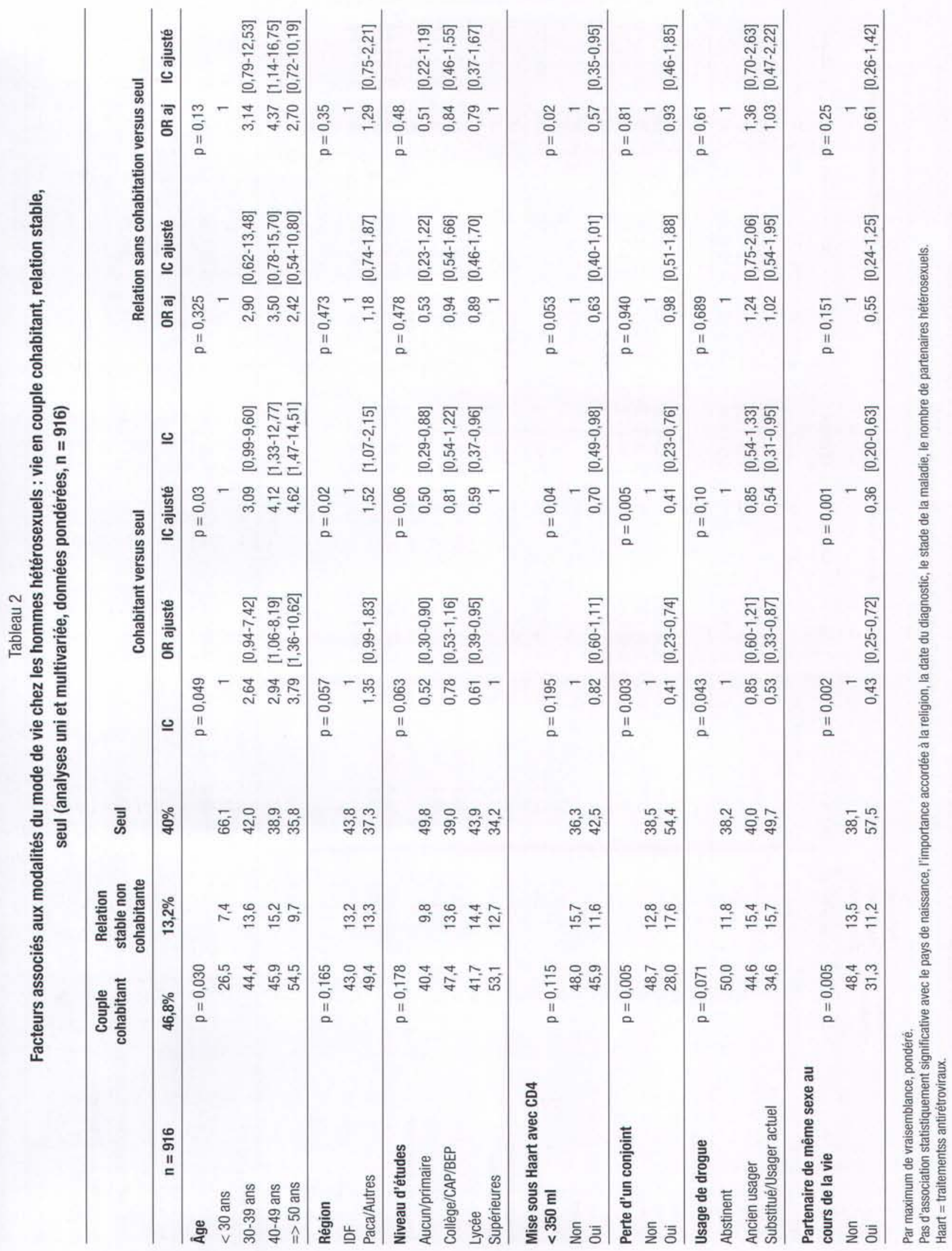


2000, chez celles qui ont eu le sida. Par contre, le fait d'avoir eu au moins une partenaire femme augmente la probabilité d'avoir une relation de couple avec un homme sans cohabiter. Toutes ces relations statistiques sont maintenues dans l'analyse multivariée (tableau 1).

Chez les hommes hétérosexuels (tableau 2), la proportion de vie en couple augmente avec l'âge, tandis que l'existence d'une relation stable non cohabitante suit une courbe en $U$, inversée en fonction de l'âge. En analyse univariée, on observe une association entre le fait de vivre en couple cohabitant et la région de résidence (plus de couples en régions), ainsi qu'avec le niveau d'étude (moins de vie en couple chez les hommes de bas niveau d'étude). La perte d'une conjointe du sida, la poursuite de l'usage de drogue ou d'un traitement de substitution et l'existence de relations homosexuelles au cours de la vie sont associées à une moindre cohabitation. En analyse multivariée, l'association forte avec l'âge se maintient, ainsi que les autres associations (région, éducation, usage de drogue, homosexualité). Les hommes hétérosexuels ayant eu un épisode grave au cours de leur maladie à VIH tendent à être plus souvent seuls, sans relation de couple.

Chez les homosexuels (tableau 3), on observe une augmentation régulière avec l'âge d'hommes vivant seuls, une proportion fluctuante de vie en cohabitation et aucune différence avec l'âge pour la catégorie relation stable sans cohabitation.

Dans ce groupe, aucune variable ne distingue les hommes seuls et les hommes avec un partenaire stable sans cohabitation. La cohabitation est associée au niveau d'étude : elle est plus fréquente chez ceux qui ont un niveau baccalauréat, plus rare chez les hommes qui accordent beaucoup d'importance à la religion et inversement proportionnelle au nombre de partenaires masculins au cours de la vie. Ces associations sont maintenues dans le modèle multivarié. Être originaire d'un autre pays européen par rapport à une naissance en France est lié au fait de vivre en couple. Ni l'ancienneté, ni la gravité de la maladie ne sont associées chez les homosexuels au fait d'avoir ou non une relation de couple au moment de l'enquête.

Les bisexuels se répartissent en $48,3 \%$ d'hommes seuls, $37,4 \%$ de cohabitants dont quatre sur cinq avec une femme, $14,3 \%$ en couples non cohabitants, dont la moitié de même sexe. Les analyses descriptives (non montrées) ne mettent pas en évidence de différence dans la répartition des variables démographiques (on retrouve seulement plus d'hommes seuls en île-de-France que dans les autres régions), sociales ou caractérisant la maladie, en fonction de la vie de couple.

Une question portait sur le rôle de l'infection par le VIH dans la dernière rupture. Celui-ci est plus souvent cité par les hommes hétérosexuels, les hommes bisexuels et les femmes que par les homosexuels masculins $(21 \%$ des femmes, $18 \%$ des hommes hétéro ou bisexuels versus $10 \%$ chez les homosexuels). Chez les femmes, la dissolution du couple est plus souvent due à la mort du conjoint ( $17 \%$ contre 2 à $6 \%$ chez les hommes).

\section{Discussion}

Les données de l'enquête Vespa décrivent la situation à l'égard de deux formes de relation stable parmi les personnes vivant avec le VIH en France en 2003. L'enquête se situe à un moment différent de la trajectoire des individus dans la maladie, selon que le diagnostic est ancien ou récent, qu'il a été fait avant ou après l'arrivée des traitements antirétroviraux hautement actifs. Le diagnostic est en effet une césure biographique dont Janine Pierret montre qu'elle entraine des réajustements différents selon que les individus se battent pour maintenir la vie " d'avant ", en font un point de rupture, à partir duquel une vie nouvelle se construit ou encore que la découverte de l'infection vient s'inscrire dans une continuité de malheurs et de difficultés. Pour tous, montre-telle, l'amour et la sexualité sont un projet et une ressource pour une vie "normale ", mais ils manquent le plus à ceux qui sont enfermés dans une spirale de malheur.

L'information rétrospective sur quelques repères chronologiques obtenue dans l'enquête Vespa ne permet pas de rétablir une histoire suffisamment fiable des différents événements de la vie affective, sociale ou médicale dans la trajectoire des individus. S'ajoute, pour les immigrés, leur parcours migratoire. Seule une approche prospective, associée à des entretiens répétés, permettrait de caractériser les trajectoires et de saisir la séquence exacte des événements, par exemple celle d'une aggravation de la maladie et d'une rupture avec le partenaire, ou de la reprise d'un emploi et d'une nouvelle relation. Ici, les seuls repères considérés sont ceux qui ont trait à l'infection à VIH : la date du diagnostic, la survenue (non datée) du sida et de la mort d'un conjoint.

L'infection par le VIH constitue, pour les personnes concernées, une "limite irréductible» $[1,2]$. Parmi les personnes hétérosexuelles vivant avec le $\mathrm{VIH}$, le taux de vie en couple est très inférieur, à âge égal, à celui de la population générale enquêtée en 1992 [9], aussi bien chez les hommes que chez les femmes: environ $60 \%$ chez les hommes et les femmes infectés par le VIH versus plus de $80 \%$ en population générale.

L'infection a un impact différent pour les homosexuels, les hommes hétérosexuels ou les femmes. En effet, la mort du conjoint/ compagnon du sida est un facteur important d'absence de vie de couple chez les hommes et les femmes hétérosexuels. Chez les homosexuels masculins à l'inverse, le fait d'avoir perdu un conjoint dans le passé n'influence pas la reconstitution d'une relation de couple. Chez les hétérosexuels, l'épidémiologie du VIH, avec une proportion majoritaire d'hommes en raison de l'importance des contaminations par usage de drogue, se traduit par le fait que les hommes séropositifs ont moins souvent une conjointe/compagne infectée (dans les couples cohabitants) : $19 \%$ de partenaires femmesVIH+ versus $29 \%$ de partenairesmasculins VIH+. Ilssontdonc moins confrontés à la perte précoce de leur partenaire (respectivement $9,2 \%$ des hommes et $19,1 \%$ des femmes ont perdu un conjoint du sida). Si les hommes hétérosexuels ont moins souvent 


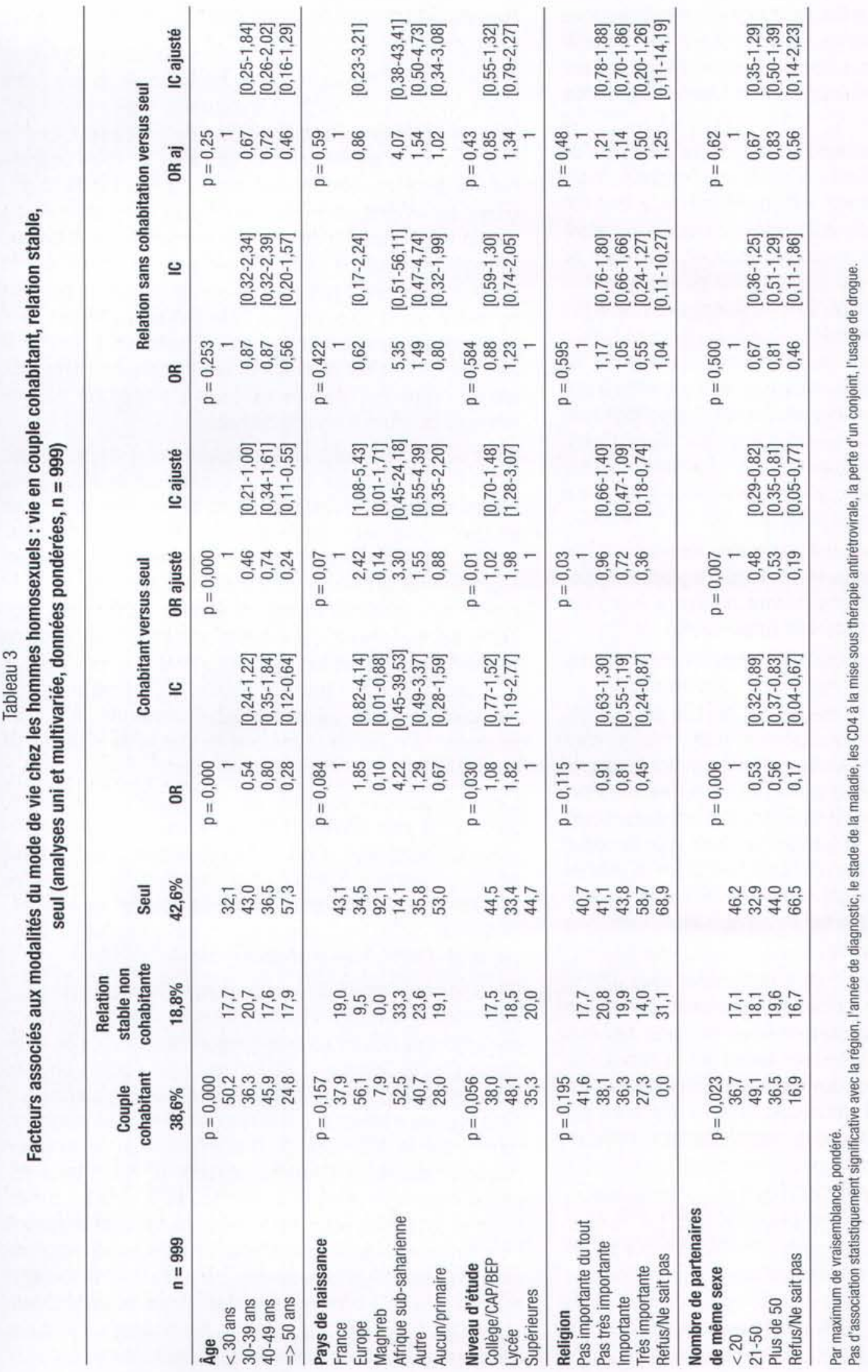


perdu leur partenaire, le deuil a les mêmes conséquences dans les deux sexes en termes de non-reconstitution des unions. En revanche, chez les hommes comme chez les femmes, l'existence d'une relation stable sans cohabitation au moment de l'enquête est statistiquement liée à la perte d'un conjoint du sida. Ce résultat associé au précédent peuts'interpréter de la façon suivante :après la mort d'un partenaire, hommes et femmes hétérosexuels tentent de reconstituer des unions, mais celles-ci ont plus de difficultés à se traduire par une cohabitation.

La majorité des situations d'absence de relation de couple est cependant principalement liée à des ruptures. Le rejet ou son anticipation, la perception de soi comme une personne non désirable ou dangereuse, la difficulté à accomplir les gestes amoureux après l'annonce du diagnostic, l'érosion de la spontanéité et du désir avec l'imposition de la protection ont été décrits par de nombreux auteurs [1,10-12]. Ces processus psychiques et intersubjectifs ne sont pas abordés par des études quantitatives telles que l'enquête Vespa. Les données étudiées suggèrent chez les hommes et les femmes hétérosexuels, et non chez les hommes homo ou bisexuels, un lien entre vie de couple et progression de la maladie : l'existence d'épisodes graves réduirait le maintien ou la reconstruction d'une relation de couple. En revanche, la durée écoulée depuis le diagnostic ne jouerait pas. Les mécanismes de ce phénomène restent à décrire : séparation du couple lors d'une maladie grave, désinvestissement de la sexualité, détérioration de l'estime de soi etc. En revanche, chez les homosexuels masculins séropositifs, la sphère de la vie sexuelle semble en quelque sorte autonomisée de l'infection par le VIH : en effet, il n'apparaît d'association ni avec les divers indicateurs de gravité de la maladie à VIH, ni avec la mort d'un conjoint/compagnon du sida. L'absence de relation de couple peut être interprétée comme un élément du style de vie. Même si les couples homosexuels sont rarement exclusifs [4], la relation de couple semble s'inscrire dans un choix qui se distingue d'un style de vie marqué par la multiplication des partenaires.

Pour les hommes et femmes hétérosexuels, le fait d'avoir eu des relations homosexuelles semble avoir une certaine spécificité en termes de trajectoire affective et sexuelle. Ainsi, chez les hommes, les rapports entre hommes au cours de la vie, qui concernent près d'un homme sur dix $(9,5 \%)$, sont associés au fait de vivre moins en couple cohabitant, à l'inverse de ceux qui n'ont pas eu ces relations $(O R=0,36$; IC $95 \%[0,20-0,63])$, alors que chez les femmes qui se déclarent hétérosexuelles, le fait d'avoir eu des partenaires féminins $(7,1 \%)$ augmente la probabilité d'une relation de couple sans cohabitation ( $O R=3,15$; IC $95 \%[1,49-6,65])$. La norme hétérosexuelle en faveur du couple semble donc s'exprimer différemment chez les hommes et chez les femmes vivant avec le VIH après des relations avec des personnes de même sexe.

Le rôle différent de l'âge chez les hommes et chez les femmes hétérosexuels correspond à ce que l'on observe en population générale : les femmes, après 35 ans, retrouvent moins une vie amoureuse et sexuelle. Elles sont plus rapidement mises à l'écart des rencontres : " l'âge a un sexe » montrent Beltzer et Bozon [13].

La poursuite de la consommation de drogue et/ou le fait de suivre un traitement de substitution ( $5 \%$ des femmes, $11,4 \%$ des hommes), regroupés ici en une seule catégorie, semblent faire obstacle à la vie de couple au cours de l'infection par le VIH. Pourtant, les traitements de substitution apportent une réelle stabilisation de la situation sociale par rapport à une vie dominée par la consommation des drogues de rue. Cependant, le traitement substitutif ne permet généralement pas une redéfinition de soi et continue à marquer l'identité [14]. II faut noter que ce groupe d'usagers et de substitués se distingue des ex-usagers, sortis de la drogue et du traitement, semblables aux autres personnes atteintes eu égard au mode de vie. On peut faire l'hypothèse qu'au cours de la maladie, la majeure partie des usagers de drogue tournent la page de la toxicomanie et, dans une certaine mesure, rompent « la spirale des malheurs " décrite par J. Pierret qui confine les personnes séropositives dans la solitude [2].

L'origine ethnique a été envisagée dans cette analyse au sens du recensement, c'est-à-dire en référence à la notion « d'immigré $»^{5}$. Les relations observées entre l'origine et le mode de vie sont difficiles à interpréter. Après ajustement, le fait d'être un immigré d'origine européenne chez les homosexuels, la naissance en France chez les hétérosexuels hommes et femmes augmente la probabilité de vie en couple ; à l'inverse, les personnes nées en Afrique sub-saharienne vivent moins en couple, mais cette association disparaît après la prise en compte des autres variables. Ainsi, il semble que les malades immigrés, hommes ou femmes, ne sont pas plus isolés que les Français du fait de la maladie, contrairement à une opinion relativement répandue. La seule association avec une variable "culturelle" concerne les homosexuels masculins : la cohabitation en couple est moins fréquente pour ceux qui accordent une place très importante à la religion (6\% des homosexuels masculins), ce qui évoque une barrière d'ordre moral à la vie en couple homosexuel.

Les femmes vivant en en Île-de-France se déclarent plus souvent isolées que celles vivant en région. Chez les hommes, cette association n'existe que pour la cohabitation et, chez les homosexuels, il n'existe pas de différence selon les régions. On ne peut exclure des phénomènes propres à la vie avec le VIH, mais les données de population générale, au recensement de 1999, indiquent une proportion un peu plus élevée de personnes seules en Île-de-France que dans le reste du pays.

Le capital éducatif et l'activité professionnelle permettent de conserver les conditions d'une vie la plus normale possible malgré la maladie. L'activité professionnelle n'a pas été prise en compte ici, car elle est très liée aux autres caractéristiques individuelles (niveau d'étude, nationalité) et à l'état de santé [15,16]. Le niveau d'étude a été choisi comme indicateur de statut social parce qu'il

Un immigrè est une personne née ètrangère à l'étranger, quelle que soit sa citoyenneté au moment du recueil de données. 
a été en général acquis antérieurement au diagnostic. Les résultats concernant l'association avec la cohabitation en couple ne sont pas clairs: on observe plus de couples cohabitants chez les hommes hétérosexuels de niveau bac que chez les hommes ayant fait des études universitaires, une tendance similaire chez les femmes, et pas de relation pour les homosexuels. Le niveau d'étude ne différencie dans aucun groupe les personnes seules des personnes ayant un partenaire stable non cohabitant. Ainsi en situation de séropositivité, le capital éducatif ne paraît pas apporter une plus grande aisance dans la vie relationnelle et faciliter l'instauration de relations de couple.

La transmissibilité sexuelle du VIH a conduit les chercheurs à se focaliser sur les comportements et les pratiques sexuelles, en abordant la sexualité à travers le prisme du risque. Ainsi, quand ils s'intéressent à la gestion du risque, ils différencient simplement les partenaires stables et les partenaires occasionnels; l'idée sous-jacente est qu'entre partenaires stables, la séropositivité est connue et la protection négociée et que, à l'inverse, la relation avec des partenaires occasionnels est marquée par une méconnaissance mutuelle. De plus, chez les homosexuels masculins dont la sexualité a été très étudiée dans le contexte du sida, la relation avec un partenaire stable est ouverte de façon plus ou moins explicite à d'autres types de relations [4]. Notre analyse indique que ces deux types de couple, cohabitants et non cohabitants, se différencient dans tous les groupes, y compris chez les homosexuels masculins. Dans les enquêtes la déclaration « de relation stable " est le plus souvent recueillie sans indication de durée ou d'autres éléments subjectifs (par exemple, l'investissement amoureux). Elle correspond sans doute à des situations très hétérogènes, alors que la cohabitation a une matérialité qui la caractérise de façon nette. Une relation stable sans cohabitation peut être un mode de vie choisi, voire une situation de transition vers la consolidation d'un couple et la cohabitation (la trajectoire inverse étant peu probable) ou une relation moins engagée. De plus, ce processus de construction, rupture, reconstruction d'une nouvelle relation en population générale obéit à des logiques différentes selon le genre [13].

\section{Conclusion}

Les femmes et les homosexuels masculins sont de plus en plus isolés avec l'âge, surtout à partir de 50 ans. Ce résultat, banal, suggère un besoin de soutien social pour faire face à la maladie, notamment au sein des associations de personnes vivant avec le VIH.

Pour autant, les besoins propres des couples dont les deux partenaires sont affectés par la maladie (de $18 \%$ chez les hommes hétérosexuels à $36,2 \%$ chez les hommes homosexuels) mériteraient une attention particulière. Si le partage de l'expérience de la maladie peut constituer un soutien pour faire face, la présence de l'infection chez les deux membres du couple peut aussi nourrir une inquiétude accrue face à l'avenir.
Les résultats de l'enquête Vespa vont dans le sens d'une solitude précoce au cours de la vie avec le VIH. La progression de la maladie et le deuil accélèrent ce processus chez les hétérosexuels, avec une situation encore plus accentuée chez les femmes, en raison de l'épidémiologie de la maladie et des rapports de genre défavorables à la reconstitution des unions chez les femmes plus âgées.

\section{Références bibliographiques}

1. Delor $F$. Vie sexuelle des personnes atteintes, sérodiscordance et risque du sida. In ouvrage collectif. Séropositivité, vie sexuelle et risque de transmission du VIH. Paris : Anrs, collection Sciences sociales et sida, 1999, p. 85-100.

2. Pierret J. Vivre avec le VIH. Enquête de longue durée auprés des personnes infectées. Paris : PUF, collection Le lien social, 2006.

3. Adam P. Bonheur dans le ghetto ou bonheur domestique? Enquête sur l'évolution des expériences homosexuelles. Actes de la recherche en sciences sociales $1999 ; 128$ : p. $56-72$.

4. Velter A, Bouyssou-Michel A. Relations stables et comportements sexuels à risque, Enquête Anrs Presse Gay 2004. Saint-Maurice : InVS, 2004, 16 p.

5. Dray-Spira R, Guéguen A, Persoz A, Deveau C, Lert F, Delfraissy JF et al. Temporary employment, absence of stable partnership, and risk of hospitalization or death during the course of HIV infection. JAIDS 2005; 40(2):190-197.

6. Young J, De Geest S, Spirig R, Flepp M, Rickenbach M, Furrer $\mathrm{H}$ et al. Stable partnership and progression to aids of death in HIV infected patients receiving highly active antiretroviral therapy: Swiss HIV cohort study. BMJ 2004; $328(7430): 15$

7. Peretti-Watel P, Riandey B, Dray-Spira R, Bouhnik AD, Sitta R, Obadia Y et I'équipe de l'enquête VESPA. Comment enquêter la population séropositive en France: l'enquête Anrs-EN12-Vespa 2003. Population 2005 ; 60(4) : 525-550.

8. Dray-Spira R, Spire B, Heard I, LertF, and the Vespa Study Group. Heterogeneous response to HAART across a diverse population of people living with HIV : results from the Anrs-EN12-Vespa Study. AIDS 2007 ; 21(S1) : S5-S12.

9. Gagnon JH, Giami A, Michaels S, de Colomby P. A comparative study of the couple in the social organization of sexuality in France and the United States. The Journal of Sex Research $2001 ; 38(1): 24-34$.

10. Meystre-Agustoni G, Thomas R, Häusermann M, Chollet-Bornand A, DuboisArber F, Spencer B. La sexualité des personnes vivant avec le VIH/sida. Lausanne: Institut universitaire de médecine sociale et préventive, raisons de santé, 17, 1998 .

11. Schiltz MA, Sandfort TG. HIV-positive people, risk and sexual behaviour. Social Science and Medicine 2000 ; 50(11): 1571-1588.

12. Mendès-Leite R, Banens M. Vivre avec le VIH. Paris : Calmann-Lévy, 2006.

13. Beltzer N, Bozon M. La vie sexuelle après une rupture conjugale. Les femmes et la contrainte de l'âge. Population $2006 ; 61(4)$ : 533-552.

14. Guichard A, Lert F, Brodeur JM, Richard L. Rapports des usagers au Subutex : de la reconquête de l'autonomie à la spirale de l'échec. Sciences Sociales et Santé $2006 ; 24(4): 5-43$.

15. Dray-Spira R, Guéguen A, Ravaud JF, Lert F and the Vespa Study Group: Socioeconomic differences in the impact of infection on workforce participation in France in the era of highly active antiretroviral therapy. American Journal of Public Health 2007 ; 97 (3) : 552-558.

16. Dray-Spira R, Lert F, and the Vespa Study Group : Living and working with HIV in France in 2003: results from the Anrs-EN12-Vespa Study. AIDS 2007; 21, suppl 1 : S29-S36. 